\title{
Evaluation of changes in left ventricular structure and function in hypertensive patients with coronary artery disease after PCI using real-time three-dimensional echocardiography
}

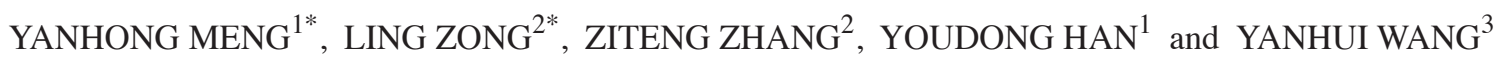 \\ Departments of ${ }^{1}$ Ultrasound, ${ }^{2}$ Thoracic Surgery and ${ }^{3}$ Imaging Center, The Affiliated Hospital \\ of Jining Medical University, Jining, Shandong 272029, P.R. China
}

Received July 25, 2017; Accepted November 1, 2017

DOI: $10.3892 / \mathrm{etm} .2017 .5544$

\begin{abstract}
We aimed to evaluate the changes in left ventricular structure and function in hypertensive patients with coronary artery disease before and after percutaneous coronary intervention (PCI) using real-time three-dimensional echocardiography. Two hundred and eighty hypertensive patients with coronary artery disease undergoing PCI and 120 cases who did not receive PCI in our hospital were selected as the subjects of our study. All patients were administered with routine antiplatelet, anticoagulant, lipid-lowering, antihypertensive, dilating coronary artery and other medications. The left ventricular systolic function and systolic synchrony index changes before and after subjects were treated by PCI were analyzed using three-dimensional echocardiography. At 2 days before surgery, there were no significant differences in the left ventricular end-diastolic volume, left ventricular endsystolic volume (LVESV) and ejection fraction (EF) between the two patient groups $(\mathrm{P}>0.05)$. At 3 months and 9 months, the two key time points after PCI, the LVESV level in the PCI group was distinctly decreased, while EF was significantly increased $(\mathrm{P}<0.05)$. In addition, before treatment, there were no significant differences in the parameters of time from the corresponding segment of the myocardium to the minimal systolic volume in two patient groups, such as Tmsv-16SD, Tmsv-16Dif, Tmsv-12SD, Tmsv-12Dif, Tmsv-6SD and Tmsv-6Dif ( $\mathrm{P}>0.05)$; however, the parameters of time from the corresponding segment of the myocardium to the minimal systolic volume in patients in the PCI group were significantly
\end{abstract}

Correspondence to: Dr Ziteng Zhang, Department of Thoracic Surgery, The Affiliated Hospital of Jining Medical University, 89 Guhuai Road, Rencheng, Jining, Shandong 272029, P.R. China E-mail: ggtgyx@163.com

*Contributed equally

Key words: essential hypertension, three-dimensional echocardiography, percutaneous coronary intervention, PCI, left ventricular systolic function, left ventricular systolic synchrony reduced at 3 and 9 months after surgery $(\mathrm{P}<0.05)$. Threedimensional echocardiography can evaluate the critical parameters in the prognosis of hypertensive patients with coronary artery disease after PCI accurately and in real-time, which may play a significant role.

\section{Introduction}

Essential hypertension is a common disease in the field of cardiology in clinical practice at present, and causal factors include mainly genetic, dietary and mental factors (1-3). The main risk for essential hypertension is the persistent elevation in blood pressure, thus increasing the left ventricular burden in patients (4-6). Recent research indicates that, with the development of the disease, cardiac structure and function in patients change significantly; in particular, the change is more obvious in hypertensive patients with coronary artery disease, and the fatality rate is clinically higher (7-9). For these patients, percutaneous coronary intervention (PCI) is the most clinically effective therapy, which can effectively restore myocardial perfusion, ameliorate systolic synchrony and cardiac function (10). When using PCI it is important to evaluate ventricular structure and function in hypertensive patients with coronary artery disease accurately and in realtime, to assist doctors to accurately assess the degree of illness, evaluate the effect of surgery, adjust medication regimens and assess patient prognosis.

Echocardiography is a non-invasive method for the diagnosis and follow-up of patients with hypertension with coronary heart disease, and the emergence of multiple new technologies makes echocardiography more widely applied $(11,12)$. Real-time three-dimensional echocardiography (RT-3DU) is the latest technology used clinically. It is based on two-dimensional ultrasound technology, and it binds with the most advanced scanning technology to increase the effect of the $\mathrm{z}$-axis direction and combines with the calculation and statistics of parameters and comparative analysis via three-dimensional parameter database to obtain accurate three-dimensional echocardiography, thus displaying cardiac conditions in patients in real-time $(13,14)$. Additionally, real-time three-dimensional echocardiography can more accurately and directly evaluate changes in 
ventricular structure and heart function of patients, including heart volume, myocardial contraction, heart function and other important parameters, thus reducing the reliance on other auxiliary means (15). RT-3DU has the advantages of rapid imaging and easy operation, which can multi-directionally display three-dimensional structure of plaque, spatial relationship and activity under the impact of blood flow, thus providing more information for clinical diagnosis (16). This study aimed to evaluate changes in left ventricular structure and function in hypertensive patients with coronary artery disease after PCI by real-time three-dimensional echocardiography, which has great research value in improving the quality of clinical PCI surgery, and to provide a theoretical basis for the wide development and application of real-time three-dimensional echocardiography.

\section{Patients and methods}

General data. Two hundred and eighty hypertensive patients with coronary artery disease undergoing PCI in our hospital from May 2014 to May 2017 were enrolled and screened in the present study, while 120 cases with the same illness that did not receive PCI were enrolled as the control group. Patients were assessed by real-time three-dimensional echocardiography at preoperative day 2, postoperative 3 months and 9 months in our study.

Inclusion criteria. Hypertensive patients with coronary artery disease, aged 50-80 years, with body mass index (BMI) within $19-25 \mathrm{~kg} / \mathrm{m}^{2}$, were included.

Exclusion criteria. The following patients were excluded: i) patients with A, B, C, D or E hepatitis virus; ii) patients with human immunodeficiency virus infection; iii) patients with other malignant tumors; iv) patients with autoimmune diseases; v) patients with cardiac structure abnormalities; vi) patients with a variety of mental disorders; vii) patients with renal insufficiency; viii) patients with hepatic insufficiency; ix) patients with other heart diseases; $x$ ) patients with other cardiac insufficiencies.

Causes for patient loss and countermeasures. The reasons for patient loss included: i) patients withdrew from this series of tests; ii) patients transferred to other hospitals for treatment during the testing; iii) patients no longer met the test conditions due to their physical condition. Therefore, the remaining subjects were supplemented into the corresponding test group in a proportion of 1:1.

Ethical considerations. Patients and lineal relatives signed an inform consent form to participate in this clinical study after fully understanding the research process. The related diagnosis, treatment and monitoring measures for patients were in accordance with the related principles of clinical guidelines, so as to fully guarantee the medical treatment and safety of the patients. The information and medical records of patients enrolled in this study were kept anonymous to protect the privacy of patients. The test program was registered in the Chinese Clinical Trial Registry, and Ottawa Group Statement for Clinical Trial Registration was followed in this test.
Strict double-blind principle. The researchers were divided into four groups: The first group of researchers was responsible for screening the test subjects that were randomly divided into two groups. The second group of researchers was responsible for the surgery in the PCI group. The third group of researchers was responsible for recording real-time threedimensional echocardiography at different time points and collecting other related data. The fourth group of researchers was responsible for collecting data, statistics and analysis, and preparation of the manuscript. The grouping of the test subjects was kept strictly anonymous. The test subjects and researchers who were responsible for clinical analysis and data collection did not know the specific grouping of the test subjects, so as to ensure that the detection and data were without human interference. Researchers in the four study groups remained anonymous for each operation.

Baseline conditions of subjects. The age, smoking history, family history, serum creatinine, homocysteine, high-sensitivity C-reactive protein (hs-CRP), blood glucose, clinical medication and other baseline data of the enrolled patients are shown in Table I.

Image collection of real-time three-dimensional echocardiography. Patients were assessed by real-time three-dimensional echocardiography using color Doppler ultrasonic diagnostic apparatus (iE33; Philips Healthcare, Andover, MA, USA). The changes in echocardiography were recorded synchronously during the determination. Patients were guided to take the left lateral position with quiet breathing. The three-dimensional probe of the apparatus was placed at the apex of the patient, and the probe angle was adjusted according to the feedback information of the image to obtain the best real-time three-dimensional image. Four cardiac cycles of real-time three-dimensional images were recorded, and the image parameters and related data were transferred to a workstation for offline analysis (Fig. 1).

Image processing and analysis. After working offline, QLAB8.1 quantitative analysis software in the workstation was opened. According to the ventricular conditions of patients, the appropriate analysis plug-in was selected to obtain the optimum results of the quantitative analysis. The important parameters of the left ventricle were mainly analyzed, including left ventricular end-systolic volume (LVESV), left ventricular ejection fraction (LVEF); the maximum difference and standard deviation of time from six middle segments of the myocardium and six basal ones to the minimum systolic volume were recorded as Tmsv-12Dif and Tmsv-12SD; the maximum difference and standard deviation of time from sixteen segments of the myocardium to the minimal systolic volume were recorded as Tmsv-16Dif and Tmsv-16SD; the maximum difference and standard deviation of time from segment of the myocardium to the minimal systolic volume (Tmsv) and its systolic time were recorded as Tmsv-Dif and Tmsv-SD; the maximum difference and standard deviation of time from six basal segments of the myocardium to the minimal systolic volume were recorded as Tmsv-6Dif and Tmsv-6SD; left ventricular end-diastolic volume (LVEDV) and the left ventricular volume-time curves (VTC). 


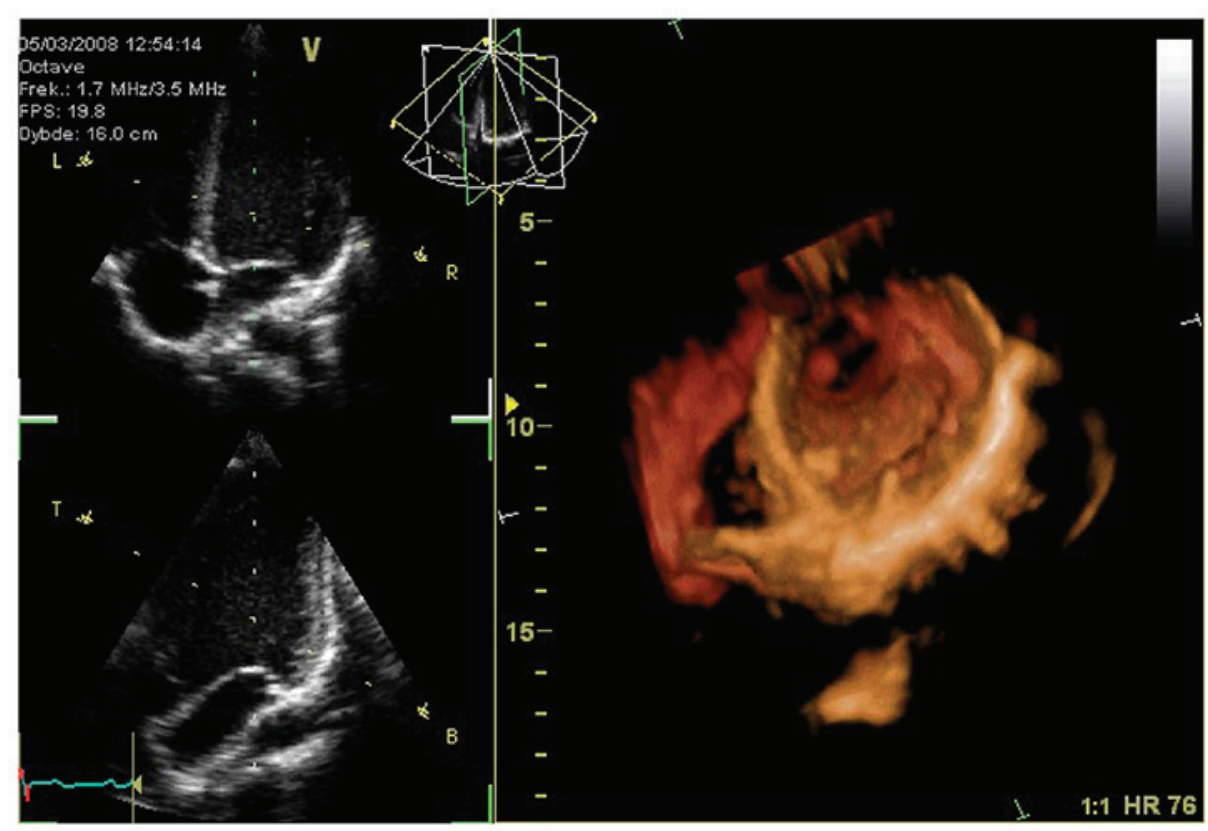

Figure 1. Real-time three-dimensional echocardiography and two-dimensional echocardiography. Left, real-time three-dimensional echocardiography; right, two-dimensional echocardiography.

Table I. Comparisons of general data of the hypertensive patients.

\begin{tabular}{|c|c|c|c|}
\hline Parameters & PCI group & Control group & P-value \\
\hline Mean age (years) & $62.47 \pm 3.82$ & $64.04 \pm 4.19$ & 0.842 \\
\hline Male, $\mathrm{n}(\%)$ & $193(68.9)$ & $84(70.0)$ & 0.747 \\
\hline Smoking, n (\%) & $104(37.1)$ & $40(33.3)$ & 0.673 \\
\hline Fasting blood-glucose (mmol/l) & $5.43 \pm 0.43$ & $5.51 \pm 0.35$ & 0.913 \\
\hline Diabetes, $\mathrm{n}(\%)$ & $79(28.2)$ & $32(26.7)$ & 0.583 \\
\hline Serum creatinine $(\mu \mathrm{mol} / \mathrm{l})$ & $92.1 \pm 9.1$ & $93.1 \pm 11.8$ & 0.738 \\
\hline Homocysteine $(\mu \mathrm{mol} / \mathrm{l})$ & $13.8 \pm 2.4$ & $13.9 \pm 1.7$ & 0.545 \\
\hline hs-CRP (mg/l) & $0.89 \pm 0.17$ & $0.91 \pm 0.21$ & 0.701 \\
\hline Aspirin, n (\%) & $280(100.0)$ & $120(100.0)$ & 0.662 \\
\hline Clopidogrel, n (\%) & $242(86.4)$ & $105(87.5)$ & 0.843 \\
\hline Ticagrelor, n (\%) & 38 (13.6) & $15(12.5)$ & 0.274 \\
\hline Statins, $\mathrm{n}(\%)$ & $276(98.6)$ & $114(95.0)$ & 0.837 \\
\hline ACEI/ARB, n (\%) & $185(66.1)$ & $81(67.5)$ & 0.583 \\
\hline$\beta$-blocker, n (\%) & $219(78.2)$ & $93(77.5)$ & 0.669 \\
\hline Calcium antagonist, $\mathrm{n}(\%)$ & $86(30.7)$ & $38(31.7)$ & 0.343 \\
\hline Nitrates, n (\%) & $251(89.6)$ & $109(90.8)$ & 0.554 \\
\hline
\end{tabular}

ACEI, angiotensin converting enzyme inhibitor; ARB, angiotensin receptor antagonist.

Clinical biochemical parameters. In addition to real-time three-dimensional echocardiography data, systolic blood pressure $(\mathrm{mmHg})$, diastolic blood pressure $(\mathrm{mmHg})$, hemoglobin $(\mathrm{g} / \mathrm{dl})$, hematocrit $(\%)$ and other clinical biochemical parameters in patients were detected.

Statistical analysis. SPSS 20.0 software (SPSS, Inc., Armonk, NY, USA) was used to statistically analyze the data for detection and observation. The Chi-square test was adopted for enumeration data, and analysis of variance (ANOVA) was utilized for measurement data, which are expressed as mean \pm standard deviation. $\mathrm{P}<0.05$ or $\mathrm{P}<0.01$ were indicative of statistically significant differences.

\section{Results}

Changes in left ventricular volume, ejection and systolic function at different time points in the hypertensive patients 
Table II. Changes in left ventricular volume, ejection and systolic function at different time points in patients receiving PCI.

\begin{tabular}{|c|c|c|c|c|}
\hline Parameters & Test time & Control group $(n=120)$ & PCI group $(n=280)$ & P-value \\
\hline \multirow{3}{*}{ LVEDV (ml) } & 2 days before treatment & $92.41 \pm 8.74$ & $92.08 \pm 7.09$ & 0.311 \\
\hline & 3 months after treatment & $92.05 \pm 8.32$ & $92.54 \pm 5.91$ & 0.284 \\
\hline & 9 months after treatment & $92.05 \pm 6.71$ & $92.07 \pm 8.22$ & 0.536 \\
\hline \multirow[t]{3}{*}{ LVESV (ml) } & 2 days before treatment & $49.28 \pm 4.92$ & $48.93 \pm 5.01$ & 0.723 \\
\hline & 3 months after treatment & $53.06 \pm 7.04$ & $47.83 \pm 6.61$ & 0.015 \\
\hline & 9 months after treatment & $49.33 \pm 4.29$ & $44.11 \pm 9.72$ & 0.013 \\
\hline \multirow[t]{3}{*}{ LVEF $(\%)$} & 2 days before treatment & $46.05 \pm 3.21$ & $46.04 \pm 3.92$ & 0.542 \\
\hline & 3 months after treatment & $45.94 \pm 3.19$ & $49.25 \pm 3.86$ & 0.006 \\
\hline & 9 months after treatment & $46.38 \pm 4.33$ & $51.74 \pm 3.22$ & 0.011 \\
\hline
\end{tabular}

Table III. Changes in left ventricular systolic volume and systolic function at different time points in hypertensive patients with coronary artery disease receiving PCI.

\begin{tabular}{|c|c|c|c|c|}
\hline Parameters & Test time & Control group $(n=120)$ & PCI group $(n=280)$ & P-value \\
\hline Tmsv-16SD & \multirow[t]{6}{*}{2 days before treatment } & $49.25 \pm 14.28$ & $40.19 \pm 11.93$ & 0.662 \\
\hline Tmsv-12SD & & $43.66 \pm 5.91$ & $44.33 \pm 7.06$ & 0.187 \\
\hline Tmsv-6SD & & $43.27 \pm 8.45$ & $43.18 \pm 7.32$ & 0.243 \\
\hline Tmsv-16Dif & & $79.15 \pm 11.31$ & $80.18 \pm 12.07$ & 0.228 \\
\hline Tmsv-12Dif & & $70.43 \pm 10.03$ & $69.18 \pm 9.06$ & 0.184 \\
\hline Tmsv-6 Dif & & $59.45 \pm 8.25$ & $59.02 \pm 9.51$ & 0.394 \\
\hline Tmsv-16SD & \multirow[t]{6}{*}{3 months after treatment } & $53.73 \pm 11.02$ & $46.06 \pm 9.83$ & 0.011 \\
\hline Tmsv-12SD & & $39.21 \pm 4.22$ & $21.36 \pm 3.11$ & 0.021 \\
\hline Tmsv-6SD & & $39.42 \pm 2.19$ & $19.04 \pm 2.33$ & 0.033 \\
\hline Tmsv-16Dif & & $80.16 \pm 9.42$ & $49.33 \pm 15.15$ & 0.018 \\
\hline Tmsv-12Dif & & $70.62 \pm 7.14$ & $50.16 \pm 2.94$ & 0.019 \\
\hline Tmsv-6 Dif & & $63.42 \pm 7.01$ & $51.22 \pm 4.05$ & 0.003 \\
\hline Tmsv-16SD & \multirow[t]{6}{*}{9 months after treatment } & $49.99 \pm 3.84$ & $19.55 \pm 3.21$ & 0.014 \\
\hline Tmsv-12SD & & $39.88 \pm 3.52$ & $20.71 \pm 3.83$ & 0.031 \\
\hline Tmsv-6SD & & $39.07 \pm 5.83$ & $20.21 \pm 3.14$ & 0.008 \\
\hline Tmsv-16Dif & & $69.21 \pm 4.05$ & $51.22 \pm 5.05$ & 0.015 \\
\hline Tmsv-12Dif & & $59.03 \pm 11.52$ & $41.37 \pm 8.21$ & 0.018 \\
\hline Tmsv-6 Dif & & $60.41 \pm 9.04$ & $41.62 \pm 5.64$ & 0.009 \\
\hline
\end{tabular}

with coronary artery disease receiving PCI. Changes in left ventricular volume, ejection and systolic function in patients receiving PCI were assessed. Before PCI, there were no significant differences in LVEDV, LVESV and LVEF between the PCI and control group ( $\mathrm{P}>0.05)$. However, results at 3 and 9 months after PCI indicated that the LVESV level in patients of the PCI group was significantly reduced when compared with that of the control group $(\mathrm{P}<0.05)$. Additionally, the LVEF value in patients of the PCI group was significantly increased when compared with that of the control group $(\mathrm{P}<0.05)$ (Table II).

Changes in left ventricular systolic volume and systolic time at different time points in hypertensive patients with coronary artery disease receiving PCI. Changes in left ventricular systolic volume and systolic time in patients receiving PCI were assessed. Before PCI, there were no significant differences in the maximum difference and standard deviation of time from segment of the myocardium to the minimal systolic volume and other important parameters between the PCI and control group $(\mathrm{P}>0.05)$. However, results at 3 and 9 months after PCI indicated that the maximum difference and standard deviation of time from segment of the myocardium to the minimal systolic volume and other important parameter levels were significantly reduced when compared with those of the control group $(\mathrm{P}<0.05)$ (Table III).

Comparisons of other functional parameter levels of the left ventricular in subjects. Comparisons of other function parameter levels of the left ventricular in subjects were carried out. 
Table IV. Comparisons of other function parameters of left ventricular in subjects (mean \pm SD).

\begin{tabular}{lcccc}
\hline Group & No. of cases & GLS $(\%)$ & GLSRs $(1 / \mathrm{sec})$ & EF $(\%)$ \\
\hline PCI group & 280 & $-19.06 \pm 1.37^{\mathrm{a}}$ & $1.05 \pm 0.29^{\mathrm{a}}$ & $65.33 \pm 6.25$ \\
Control group & 120 & $-22.33 \pm 2.15$ & $1.25 \pm 0.31$ & $67.03 \pm 5.75$ \\
\hline
\end{tabular}

${ }^{\text {a }}<0.05$, compared with the control group.

Table V. Comparisons routine heart structure parameters of left ventricular in subjects (mean $\pm \mathrm{SD}$ ).

\begin{tabular}{lccccc}
\hline Group & No. of cases & LVEDD $(\mathrm{cm})$ & IVSD $(\mathrm{cm})$ & LVPWD $(\mathrm{cm})$ & LVMI $\left(\mathrm{g} / \mathrm{m}^{2}\right)$ \\
\hline PCI group & 280 & $4.86 \pm 0.37^{\mathrm{a}}$ & $1.04 \pm 0.29^{\mathrm{a}}$ & $0.94 \pm 0.26^{\mathrm{a}}$ & $65.33 \pm 6.25^{\mathrm{a}}$ \\
Control group & 120 & $4.33 \pm 0.15$ & $0.88 \pm 0.31$ & $0.78 \pm 0.33$ & $67.03 \pm 5.75$ \\
\hline
\end{tabular}

${ }^{\mathrm{a}} \mathrm{P}<0.05$, compared with the control group.

Table VI. Comparisons of clinical biochemical parameters in subjects (mean \pm SD).

\begin{tabular}{lcccr}
\hline Group & $\begin{array}{c}\text { No. of } \\
\text { cases }\end{array}$ & $\begin{array}{c}\text { Systolic blood } \\
\text { pressure }(\mathrm{mmHg})\end{array}$ & $\begin{array}{c}\text { Diastolic blood } \\
\text { pressure }(\mathrm{mmHg})\end{array}$ & $\begin{array}{c}\text { Hemoglobin } \\
(\mathrm{g} / \mathrm{dl})\end{array}$ \\
\hline PCI group & 280 & $133.6 \pm 10.7^{\mathrm{a}}$ & $76.4 \pm 9.2$ & $\begin{array}{c}\text { Hematocrit } \\
(\%)\end{array}$ \\
Control group & 120 & $124.3 \pm 9.5$ & $75.8 \pm 9.3$ & $10.78 \pm 2.6^{\mathrm{a}}$ \\
\hline
\end{tabular}

${ }^{\mathrm{a}} \mathrm{P}<0.05$, compared with the control group.

Compared with that in the control group, global longitudinal strain (GLS) (\%) level in patients of the PCI group was significantly increased $(\mathrm{P}<0.05)$. Additionally, global longitudinal systolic strain rates (GLSRs) $(1 / \mathrm{sec})$ and ejection fraction (EF) $(\%)$ level in patients of the PCI group were significantly reduced $(\mathrm{P}<0.05)$ (Table IV).

Comparisons of routine heart structure parameters of the left ventricular in subjects. Comparisons of routine heart structure parameters of left ventricular in subjects were carried out. Compared with that in the control group, the left ventricular mass index (LVMI) $\left(\mathrm{g} / \mathrm{m}^{2}\right)$ level in patients of the PCI group was significantly increased $(\mathrm{P}<0.05)$. Additionally, left ventricular end-diastolic dimension (LVEDD) (cm), interventricular septum thickness at end-diastole (IVSD) $(\mathrm{cm})$ and left ventricular posterior wall depth (LVPWD) $(\mathrm{cm})$ levels in patients of the PCI group were significantly reduced $(\mathrm{P}<0.05)$ (Table V).

Comparisons of clinical biochemical parameters in subjects. Comparisons of clinical biochemical parameters in subjects: Compared with those in the control group, systolic blood pressure $(\mathrm{mmHg})$ and hemoglobin $(\mathrm{g} / \mathrm{dl})$ level in patients of the PCI group were significantly increased $(\mathrm{P}<0.05)$. Additionally, diastolic blood pressure $(\mathrm{mmHg})$ and hematocrit $(\%)$ level in the patients of the PCI group had no distinct changes $(\mathrm{P}>0.05)$ (Table VI).

\section{Discussion}

Essential hypertension is a common disease in the field of cardiology in clinical practice at present, and its cause is not clear, but mainly includes genetic, dietary and mental factors. The main risk for essential hypertension is the persistent elevation in blood pressure, thus increasing the left ventricular burden in patients, finally incurring coronary heart disease. The routine left ventricular systolic function is measured by M-ultrasound or two-dimensional echocardiography Simpson's method with the premise of regular morphology of heart (17-19). However, in pathological conditions, such as ventricular aneurysm, ventricular wall motion abnormality and other cardiac morphological or functional abnormalities, its accuracy is significantly affected. Real-time three-dimensional echocardiography has a three-dimensional matrix probe controlled by a computer, and works by a mechanism of fast scanning via multi-directional sound beam to emit three-dimensional scanning sound beam. Due to the simultaneous scanning of sound beam in three directions perpendicular to each other, a threedimensional structural image database is formed that covers various regions of the heart with the shape of a pyramid, thus obtaining real-time three-dimensional image of the heart. In contrast, three-dimensional echocardiography can automatically analyze and obtain left ventricular volume on the basis of direct tracking of the endocardium, which overcomes the defects of geometric assumption, and the measured value is 
more close to the actual value (5). Previous studies have shown that three-dimensional echocardiography used to measure left ventricular end-diastolic volume is closer to magnetic resonance imaging (MRI) which is the gold standard to evaluate left ventricular volume, indicating that three-dimensional echocardiography is more accurate than the traditional twodimensional echocardiography (20-25).

Three-dimensional echocardiography can also provide quantitative indices of synchronous contractions in each segment of the left ventricle. The three key time points, 2 days before surgery, 3 and 9 months after surgery, were selected in this study, revealing that at 3 months after PCI, the kinetic energy of the left ventricle in patients was significantly increased. At 9 months after surgery, left ventricular function in patients basically returned to normal, which was consistent with the results of previous studies. In this study, LVEDV in hypertensive patients with coronary artery disease after PCI was reduced when compared to that before surgery, while LVESV was decreased, and LVEF was increased. Meanwhile, it was found that at day 2 before PCI, there were no significant differences in the maximum difference and standard deviation of time from segment of the myocardium to the minimal systolic volume and other important parameters between the PCI and control group. Additionally, the results at 3 and 9 months after surgery indicated that the maximum difference and standard deviation of time from segment of the myocardium to the minimal systolic volume and other important parameter levels were obviously reduced in patients in the PCI group, revealing that postoperative left ventricular function gradually returned to the normal level. Compared with the control group, the GLS (\%) level in patients of the PCI group was significantly increased, while GLSRs $(1 / \mathrm{sec})$ and EF $(\%)$ level in patients of the PCI group were significantly reduced $(\mathrm{P}<0.05)$. GLS represents the extent of the shortening of the left ventricle along the longitudinal fibrous direction of the myocardium during the systolic period. Due to the long-term effect of blood flow, fibrosis occurs, indicating that postoperative left ventricular function in patients returned to normal. Additionally, the LVMI $\left(\mathrm{g} / \mathrm{m}^{2}\right)$ level in patients of the PCI group was significantly increased $(\mathrm{P}<0.05)$, while LVEDD $(\mathrm{cm})$, IVSD $(\mathrm{cm})$ and LVPWD $(\mathrm{cm})$ levels in patients of the PCI group were significantly reduced $(\mathrm{P}<0.05)$. Therein, triglyceride is an important factor to affect the level of LVMI, representing the extent of left ventricular hypertrophy. Finally, systolic blood pressure $(\mathrm{mmHg})$ and hemoglobin $(\mathrm{g} / \mathrm{dl})$ level in the patients of the PCI group were significantly increased $(\mathrm{P}<0.05)$. Additionally, diastolic blood pressure $(\mathrm{mmHg})$ and hematocrit (\%) level in patients of the PCI group had no distinct changes $(\mathrm{P}>0.05)$. The above results indicated that left ventricular systolic synchrony and global systolic function were gradually improved after PCI.

In summary, real-time three-dimensional echocardiography can accurately and efficiently evaluate the changes in left ventricular structure and function in hypertensive patients with coronary artery disease receiving PCI, which can replace the traditional two-dimensional ultrasound. In order to further enhance the success rate of PCI and detect heart function and structural changes in patients, real-time three-dimensional echocardiography is worthy of clinical popularization and application as an effective detection means.

\section{References}

1. Brott BC: Prevention of myocardial stunning during percutaneous coronary interventions: Novel insights from pre-treatment with glucagon-like peptide-1. JACC Cardiovasc Interv 8: 302-304, 2015 .

2. Fiechter M, Fuchs TA, Stehli J, Jacobs S, Falk V and Kaufmann PA: Reversible true myocardial hibernation. Eur Heart J 34: 648, 2013.

3. Page BJ, Banas MD, Suzuki G, Weil BR, Young RF, Fallavollita JA, Palka BA and Canty JM Jr: Revascularization of chronic hibernating myocardium stimulates myocyte proliferation and partially reverses chronic adaptations to ischemia. $\mathbf{J}$ Am Coll Cardiol 65: 684-697, 2015.

4. Lang RM, Bierig M, Devereux RB, Flachskampf FA, Foster E, Pellikka PA, Picard MH, Roman MJ, Seward J, Shanewise JS, et al; Chamber Quantification Writing Group; American Society of Echocardiography's Guidelines and Standards Committee; European Association of Echocardiography: Recommendations for chamber quantification: A report from the American Society of Echocardiography's Guidelines and Standards Committee and the Chamber Quantification Writing Group, developed in conjunction with the European Association of Echocardiography, a branch of the European Society of Cardiology. J Am Soc Echocardiogr 18: 1440-1463, 2005.

5. Bayramoğlu A, Taşolar H, Otlu YÖ, Hidayet Ş, Kurt F, Doğan A and Pekdemir H: Assessment of left atrial volume and mechanical functions using real-time three-dimensional echocardiography in patients with mitral annular calcification. Anatol J Cardiol 16: 42-47, 2016.

6. Saloux E, Labombarda F, Pellissier A, Anthune B, Dugué AE, Provost N, Allain P, De Craene M, Milliez P and Manrique A: Diagnostic value of three-dimensional contrast-enhanced echocardiography for left ventricular volume and ejection fraction measurement in patients with poor acoustic windows: A comparison of echocardiography and magnetic resonance imaging. J Am Soc Echocardiogr 27: 1029-1040, 2014.

7. Aurich M, André F, Keller M, Greiner S, Hess A, Buss SJ, Katus HA and Mereles D: Assessment of left ventricular volumes with echocardiography and cardiac magnetic resonance imaging: Real-life evaluation of standard versus new semiautomatic methods. J Am Soc Echocardiogr 27: 1017-1024, 2014.

8. Hoffmann R, Barletta G, von Bardeleben S, Vanoverschelde JL, Kasprzak J, Greis C and Becher H: Analysis of left ventricular volumes and function: A multicenter comparison of cardiac magnetic resonance imaging, cine ventriculography, and unenhanced and contrast-enhanced two-dimensional and threedimensional echocardiography. J Am Soc Echocardiogr 27: 292-301, 2014.

9. Rosendahl L, Blomstrand P, Brudin L, Tödt T and Engvall JE: Longitudinal peak strain detects a smaller risk area than visual assessment of wall motion in acute myocardial infarction. Cardiovasc Ultrasound 8: 2, 2010.

10. Xiong XJ, Li SJ and Zhang YQ: Massage therapy for essential hypertension: A systematic review. J Hum Hypertens 29: 143-151, 2015.

11. Markou A, Sertedaki A, Kaltsas G, Androulakis II, Marakaki C, Pappa T, Gouli A, Papanastasiou L, Fountoulakis S, Zacharoulis A, et al: Stress-induced aldosterone hyper-secretion in a substantial subset of patients with essential hypertension. J Clin Endocrinol Metab 100: 2857-2864, 2015.

12. Yan B, Peng L, Dong Q, Zheng F, Yang P, Sun L, Gong S, Zeng L and Wang G: Reverse-dipper pattern of blood pressure may predict lacunar infarction in patients with essential hypertension. Eur J Neurol 22: 1022-1025, 2015.

13. Celık T, Balta S, Karaman M, Ahmet Ay S, Demırkol S, Ozturk C, Dinc M, Unal HU, Yilmaz MI, Kilic S, et al: Endocan, a novel marker of endothelial dysfunction in patients with essential hypertension: Comparative effects of amlodipine and valsartan. Blood Press 24: 55-60, 2015.

14. Bayramoglu A, Urhan Kucuk M, Guler HI, Abaci O, Kucukkaya Y and Colak E: Is there any genetic predisposition of MMP-9 gene C1562T and MTHFR gene C677T polymorphisms with essential hypertension? Cytotechnology 67: 115-122, 2015.

15. Wang C, Han L, Wu Q, Zhuo R, Liu K, Zhao J, Zhang L, Hao Y, Fan R, Liu Y, et al: Association between homocysteine and incidence of ischemic stroke in subjects with essential hypertension: A matched case-control study. Clin Exp Hypertens 37: $557-562,2015$. 
16. Dörr O, Liebetrau C, Möllmann H, Mahfoud F, Ewen S, Gaede L, Troidl C, Hoffmann J, Busch N, Laux G, et al: Beneficial effects of renal sympathetic denervation on cardiovascular inflammation and remodeling in essential hypertension. Clin Res Cardiol 104: $175-184,2015$.

17. Zhong F, Zhuang L, Wang Y and Ma Y: Homocysteine levels and risk of essential hypertension: A meta-analysis of published epidemiological studies. Clin Exp Hypertens 39: 160-167, 2017.

18. Wrzosek M, Sokal M, Sawicka A, Wlodarczyk M, Glowala M, Wrzosek M, Kosior M, Talalaj M, Biecek P and Nowicka G: Impact of obesity and nitric oxide synthase gene G894T polymorphism on essential hypertension. J Physiol Pharmacol 66: 681-689, 2015.

19. Zhang SY, Chan SW, Zhou X, Chen XL, Mok DK, Lin ZX and Wang YH: Meta-analysis of association between ALDH2 rs671 polymorphism and essential hypertension in Asian populations. Herz 40 (Suppl 2): 203-208, 2015.

20. Khan SG, Geer A, Fok HW, Shabeeh H, Brett SE, Shah AM and Chowienczyk PJ: Impaired neuronal nitric oxide synthasemediated vasodilator responses to mental stress in essential hypertension. Hypertension 65: 903-909, 2015.

21. Cui C, Liu L, Fan T, Peng B, Cheng Z, Ge Z, Li Y, Liu Y, Zhang Y, Ai F, et al: Application of real-time three-dimensional echocardiography to evaluate the pre- and postoperative right ventricular systolic function of patients with tetralogy of fallot. Acta Cardiol Sin 31: 345-352, 2015.
22. Yang L, Ma L, Li Y, Mu Y and Liu L: Real-time three-dimensional echocardiography of left atrial volume and function in patients with severe multi-vessel coronary artery disease. J Med Ultrason (2001) 44: 71-78, 2017.

23. Zhao H, Fu YM and Jia YM: Right heart function assessment with real-time three-dimensional echocardiography before and after atrial septal defect surgery. Hainan Yixueyuan Xuebao 22: 117-120, 2016.

24. Velasco O, Beckett MQ, James AW, Loehr MN, Lewis TG, Hassan $\mathrm{T}$ and Janardhanan R: Real-time three-dimensional echocardiography: Characterization of cardiac anatomy and function-current clinical applications and literature review update. Biores Open Access 6: 15-18, 2017.

25. Chen HY, Pan CZ and Shu XH: Partially unroofed coronary sinus diagnosed by real-time three-dimensional transesophageal echocardiography after operation of secundum atrial septal defect. Int J Cardiovasc Imaging 31: 45-46, 2015.

This work is licensed under a Creative Commons Attribution-NonCommercial-NoDerivatives 4.0 International (CC BY-NC-ND 4.0) License. 